\title{
A Monte Carlo Study of Gamma-Ray Pulsars in the Gould Belt
}

\author{
P. Leung, K. S. Cheng \\ Department of Physics, University of Hong Kong, Hong Kong, PRC \\ L. Zhang, Z. J. Jiang \\ Department of Physics, Yunnan University, Kunming, PRC
}

\begin{abstract}
We use Monte Carlo techniques to simulate the statistical properties of rotation-powered pulsars in the Gould Belt. The gammaray properties of these pulsars are calculated by using a self-consistent outer gap model and other pulsar properties, i.e., initial magnetic field and period, and velocity distribution of the neutrons stars at birth, are obtained from the statistics of radio pulsars. We obtain distributions of the magnetic inclination angle, period, distance and age for these gammaray pulsars in the Gould Belt.
\end{abstract}

\section{Introduction}

There are 170 unidentified $\gamma$-ray sources in the third EGRET catalog, with $\sim 50$ sources close to the Galactic plane with $|b|<5^{\circ}$, and $\sim 70$ sources at medium latitudes with $|b|$ between $5^{\circ}$ and $30^{\circ}$. For those unidentified $\gamma$-ray sources in the Galactic plane, many of them are associated with Wolf-Rayet and Of stars, supernova remnants and OB stars. All of these objects are considered pulsar tracers; therefore it is natural to suggest that these low latitude sources may be Geminga-like pulsars, which are radio-quiet. However, medium latitude sources have significantly softer spectra, are fainter and have a steeper $\log N$-logS function than those at low latitudes (Gehrels et al. 2000). It has been suggested that they are associated with recent supernovae in the nearby Gould Belt.

\section{The Model}

When the geometry of the dipole magnetic field is taken into account, the derived outer gap size is not only a function of period $(P)$ and magnetic field $(B)$ of the neutron star, but also a function of the average radial distance to the neutron star $\langle r\rangle$; depends on the magnetic inclination angle $(\alpha)$. The fractional size of the outer gap, $f(P, B,\langle r\rangle(\alpha))$, is used to describe the thickness of the outer gap and is determined by the $e^{ \pm}$pair production. The outer gap will not exist if $f(r, \alpha)>1$. In estimating the fractional size of the outer gap, we assume that $\mathrm{X}$-rays are produced by the bombardment of the relativistic particles from the outer gap on the stellar surface (the outer gap is called as a self-sustained outer 

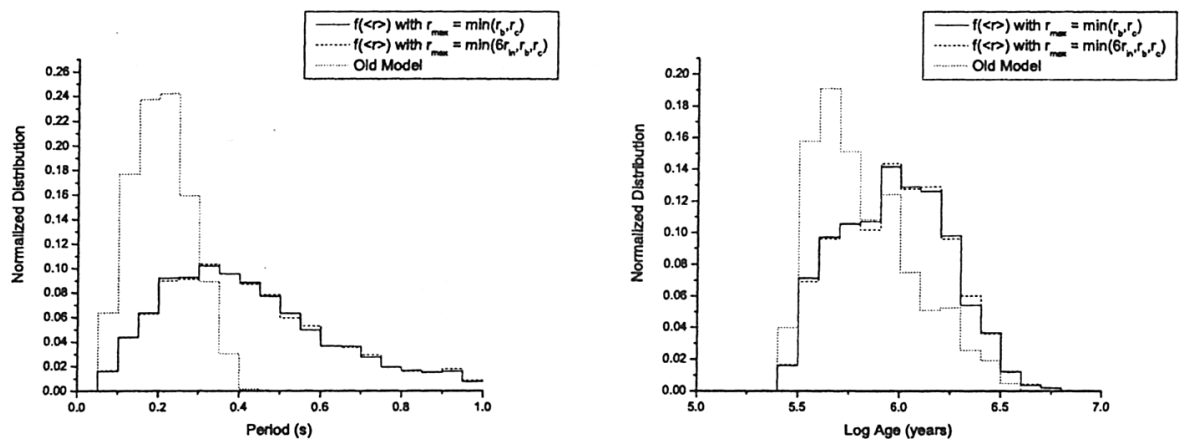

Figure 1. Period and age of simulated Gould Belt pulsars.

gap). Therefore, we have:

$$
\begin{gathered}
f(r, \alpha)=5.2 B_{12}^{-4 / 7} P^{26 / 21} R_{6}^{10 / 7} G(r, \alpha), \text { with } \\
G(r, \alpha)=\left[\frac{2}{1-\cos \theta_{X_{\gamma}}}\right]^{\frac{4}{7}}\left(\ln \frac{r}{R}\right)^{\frac{1}{21}}\left(\frac{r}{R_{L}}\right)^{\frac{13}{14}}\left(\frac{W}{a(\alpha) \cos \alpha}\right)^{\frac{1}{7}}\left(\frac{\sqrt{1+3 \cos ^{2}(\theta-\alpha)}}{2}\right)^{\frac{-3}{7}}
\end{gathered}
$$

where

$$
W(\alpha, r)=\frac{4}{3} \frac{\left[1-\frac{3}{4} a(\alpha) \frac{r}{R_{L}}\right]^{3 / 2}}{\sqrt{a(\alpha)}\left(1-\frac{1}{2} a(\alpha) \frac{r}{R_{L}}\right)} .
$$

\section{Results}

As shown in Figure 1, the simulated pulsars in the Gould Belt have a very wide range of periods, which are very different from those of the Galactic plane pulsars (Cheng \& Zhang 1998). Furthermore, their typical age is near one million years. We have simulated the properties of the gamma-ray pulsar population in the nearby Gould Belt in terms of the revised thick outer gap models and Monte Carlo methods. The initial magnetic field, spatial and velocity distributions of neutron stars at birth which are obtained from radio pulsar statistical studies have been used in our simulations. We have modeled the spatial, distance, period, age, magnetic field and energy flux distributions of this gamma-ray pulsar population. We will compare our model results with the medium latitude gamma-ray sources detected by EGRET and present it in the future.

\section{References}

Cheng, K. S., \& Zhang, L. 1998, ApJ, 498, 327

Gehrels, N., Macomb, D. J., Bertsch, D. L., Thompson, D. J., \& Hartman, R. C. 2000, Nature, 404, 363 